\title{
As diferenças de idade e gênero entre usuários de CAPS ad e as implicações na rede de atenção
}

\author{
Marcos Antonio Lopes ${ }^{1}$ \\ Jonas Salomão Sprícigo ${ }^{1}$ \\ Myriam Raquel Mitjavila ${ }^{1}$ \\ Daniela Ribeiro Schneider ${ }^{1}$ \\ Deidvid Abreu ${ }^{1}$
}

Objetivo: este estudo faz parte das atividades do Programa de Educação pelo Trabalho para a Saúde (PET-Saúde) e teve como objetivo realizar um levantamento clínico e sociodemográfico dos usuários dos Centros de Atenção Psicossocial, Álcool e Outras Drogas (CAPSs ad) de Florianópolis. Método: é um estudo observacional e transversal. Os dados foram coletados dos prontuários eletrônicos. Resultados: a população foi composta de 1.191 usuários dos CAPSs ad Continente e Ilha. No CAPS ad Ilha, as mulheres dependentes de álcool eram mais jovens do que os homens $(p=0,075)$ e, em ambos CAPS ad, os usuários de cocaína/ crack eram mais jovens do que os de álcool $(p<0,05)$. Conclusão: as diferenças etárias e de gênero têm implicações na rede de atenção.

Descritores: Álcool; Drogas; Usuários; Serviços de Saúde Mental.

\footnotetext{
${ }^{1}$ Universidade Federal de Santa Catarina, Florianópolis, SC, Brasil.
} 


\title{
Differences in age and gender among CAPS ad users and the implications in the health care network
}

Objective: this study is part of the activities of the project Programa de Educação pelo Trabalho para a Saúde (PET-Saúde) [Education through Labor for Health Program ] and aimed to carry out a clinical and sociodemographic survey of users treated in Centros de Atenção Psicossocial, Álcool e Outras Drogas (CAPSs ad) [Psychosocial Care Centers for Alcohol and Other Drugs], in the City of Florianópolis. Method: this is an observational and cross-sectional study. Data were collected through access to electronic records. Results: the population consisted of 1,191 Mainland and Island CAPS ad users. At the Island CAPS ad, alcohol dependent women were younger than men $(p=0.075)$ and, in both CAPSs ad, cocaine/crack users were younger than alcohol users $(p<0.05)$. Conclusion: the age and gender differences have implications in the health care network.

Descriptors: Alcohol; Drugs; Users; Mental Health Services.

\section{Las diferencias etarias y de género entre usuarios de CAPSs ad y las implicaciones en la red de atención}

\begin{abstract}
Objetivo: este estudio forma parte de las actividades del Programa de Educação pelo Trabalho para a Saúde (PET-Saúde) [Programa de Educación por el Trabajo para la Salud] y tuvo como objetivo realizar un relevamiento clínico y sociodemográfico de los usuarios de los Centros de Atenção Psicossocial, Álcool e Outras Drogas (CAPSs ad) [Centros de Atención Psicosocial, Alcohol y Otras Drogas], en la Ciudad de Florianópolis. Método: es un estudio observacional y transversal. Los datos fueron relevados de los registros electrónicos. Resultados: la población estuvo compuesta por 1.191 usuarios de los CAPSs ad Continente e Isla. En el CAPS ad Isla, las mujeres dependientes de alcohol eran más jóvenes que los hombres $(p=0,075)$ y, en ambos CAPSs ad, los usuarios de cocaína/crack eran más jóvenes que los usuarios de alcohol $(p<0,05)$. Conclusión: las diferencias etarias y de género tienen implicaciones en la red de atención.
\end{abstract}

Descriptores: Alcohol; Drogas; Usuarios; Servicios de Salud Mental.

\section{Introdução}

Os Centros de Atenção Psicossocial (CAPSs) vêm se consolidando como importantes dispositivos para o cuidado em saúde mental para a população brasileira. Desde a sua criação, por intermédio da Portaria $n^{\circ} 336 /$ $\mathrm{GM}$, de 19 de fevereiro de $2002^{(1)}$, que estabeleceu as várias modalidades de CAPS destinadas prioritariamente ao atendimento de usuários com transtornos mentais graves, 1.620 unidades foram implantadas até o ano de 2010 em todas as regiões do país ${ }^{(2)}$. Essa expansão seguiu uma trajetória dos grandes para os pequenos centros urbanos e teve um forte incremento na região Nordeste, que se posicionou, ao final deste período, como a segunda região em cobertura proporcional de CAPSs $(0,81 / 100$ mil habitantes $)$, atrás apenas da região Sul $(0,87 / 100$ mil habitantes). Em especial, há que se destacar também o aumento de $87 \%$ na oferta de Centros de Atenção Psicossocial, Álcool e Outras Drogas (CAPSs ad) no período de 2006 a 2010, totalizando 258 unidades destinadas ao atendimento 
de usuários com problemas relacionados ao uso de álcool e outras drogas.

Do ponto de vista funcional, segundo levantamento realizado com 184 coordenadores de CAPSs ad, 75\% dos serviços contavam com equipe multiprofissional completa e quase a totalidade oferecia uma boa diversidade de atendimentos - familiar, em grupo, oficina e visita domiciliar(2). Esses dados apontam, então, que os CAPSs ad que figuram dentro da Rede de Atenção Psicossocial( ${ }^{(3)}$ como os principais pontos de atenção psicossocial aos usuários de álcool e outras drogas apresentavam as condições apropriadas para o atendimento das políticas públicas que já haviam sido estabelecias e vêm se solidificando nessa área. Tanto a Política de Atenção Integral ao Usuário de Álcool e Drogas $^{(4)}$ quanto o Plano Integrado de Enfrentamento ao Crack e outras Drogas ${ }^{(5)}$ priorizam a integralidade e a transversalidade do cuidado ao usuário, encontrando, dessa forma, sintonia com a realidade existente nos CAPSs ad. Ademais, tais políticas têm entre seus objetivos o desenvolvimento de recursos humanos e a realização de estudos e diagnósticos que contribuam para o aperfeiçoamento das medidas de prevenção do uso, tratamento e reinserção social do usuário.

Em relação ao perfil dos usuários em atendimento, os dados produzidos pelo levantamento com os coordenadores de CAPSs ad revelaram uma população predominantemente de adultos e jovens, que usam principalmente álcool, crack e tabaco. Outros três estudos brasileiros também exibiram dados sociodemográficos e de padrão de uso de substâncias entre os usuários de CAPSs ad. O estudo de Feira de Santana (BA) comparou adolescentes (12 a 19 anos) e adultos jovens (20 a 24 anos) e observou maior proporção de uso pesado de maconha entre os adolescentes e de uso pesado de álcool entre os adultos jovens ${ }^{(6)}$. Examinando apenas os usuários de álcool, o estudo de Teresina (PI) observou quase metade de sua amostra com emprego, estado civil casado e escolaridade de ensino fundamental, sendo que, em relação ao padrão de uso, quase a totalidade fazia uso diariamente $(55 \%)$ ou acima de três vezes por semana $(35,2 \%)^{(7)}$. No estudo de Blumenau (SC), o predomínio ainda era dos problemas decorrentes do uso de álcool, seguido do uso cruzado de substâncias, principalmente o tripé álcool/maconha/ cocaína-crack. O uso de álcool era maior conforme aumentava a idade, enquanto o uso cruzado percorria um caminho inverso, com maior ocorrência entre os mais jovens ${ }^{(8)}$. Em todos os estudos, a população era predominantemente masculina (acima de 85\%).

Um ponto que precisa ser investigado é a representação nos CAPSs ad em relação à população de usuários de álcool e outras drogas presentes na comunidade. Se considerarmos o uso no último mês ${ }^{(9)}$, que seria o mais próximo do uso atual e presente, quais proporções dos $36,1 \%$ de usuários de álcool, 0,3\% de usuários de maconha e 0,3\% de usuários de cocaína estão em atendimento nos CAPSs ad? O predomínio de uso de álcool entre os homens, o pico de uso de álcool nos homens na faixa entre 25 a 34 anos e o pico nas mulheres entre 18 a 24 anos de alguma maneira se reproduzem nos CAPSs ad? Em suma, faz-se necessária a obtenção de informações sobre a clientela que demanda atendimento nos CAPSs ad, buscando assim um melhor entendimento sobre o papel que esse dispositivo ocupa junto à população e à rede de atenção. O presente estudo tem como objetivos realizar um levantamento sociodemográfico e clínico dos usuários assistidos nos CAPSs ad do Município de Florianópolis (SC), investigar a prevalência dos principais diagnósticos psiquiátricos na população examinada com ênfase nos relacionados ao uso de substâncias psicoativas e examinar a distribuição sociodemográfica e clínica destes diagnósticos.

\section{Métodos}

Este estudo faz parte das atividades do projeto intitulado Programa de Educação pelo Trabalho para a Saúde - PET-Saúde/Saúde Mental - Crack, Álcool e Outras Drogas (PET-Saúde/Saúde Mental/Crack), promovido pelos Ministérios da Saúde (Secretaria de Gestão do Trabalho e da Educação na Saúde, Secretaria de Atenção à Saúde e Secretaria Nacional de Políticas sobre Drogas) e da Educação (Secretaria de Educação Superior)(10). "O PET-Saúde/Saúde Mental/ Crack tem como pressuposto a educação pelo trabalho e é destinado a fomentar grupos de aprendizagem tutorial no âmbito da Atenção em Saúde Mental, Crack, Álcool e Outras Drogas, caracterizando-se como instrumento para qualificação em serviço dos profissionais para a atenção em saúde mental, crack, álcool e outras drogas, bem como de iniciação ao trabalho e formação dos estudantes dos cursos de graduação da área da saúde, de acordo com as necessidades do Sistema Único de Saúde (SUS), tendo em perspectiva a qualificação da atenção e a inserção das necessidades dos serviços como fonte de produção de conhecimento e pesquisa nas instituições de ensino superior". Este projeto foi executado pela parceria entre a Universidade Federal de Santa Catarina (UFSC) e a Prefeitura Municipal de Florianópolis (PMF) e contou com a participação de quatro professores e 24 estudantes da UFSC e quatro técnicos da PMF, no período de março de 2011 a fevereiro de 2012. 
Tabela 1 - Distribuição sociodemográfica da amostra nos dois CAPSs ad (Continente e llha). Florianópolis, SC, Brasil, 2012

\begin{tabular}{|c|c|c|c|c|c|c|}
\hline \multirow[t]{2}{*}{ Local/Variável } & \multicolumn{2}{|c|}{ Feminino } & \multicolumn{2}{|c|}{ Masculino } & \multicolumn{2}{|c|}{ Total } \\
\hline & $\mathrm{n}$ & $\%$ & $\mathrm{n}$ & $\%$ & $\mathrm{n}$ & $\%$ \\
\hline \multicolumn{7}{|l|}{ CAPS ad Continente } \\
\hline \multicolumn{7}{|l|}{ Idade } \\
\hline 0 a 9 & 0 & 0 & 0 & 0 & 0 & 0 \\
\hline 10 a 19 & 5 & 5,4 & 19 & 4,3 & 24 & 4,5 \\
\hline 20 a 29 & 15 & 16,1 & 106 & 24,0 & 121 & 22,6 \\
\hline 30 a 39 & 25 & 26,9 & 131 & 29,6 & 156 & 29,2 \\
\hline 40 a 49 & 24 & 25,8 & 113 & 25,6 & 137 & 25,6 \\
\hline 50 a 59 & 15 & 16,1 & 55 & 12,4 & 70 & 13,1 \\
\hline 60 a 69 & 5 & 5,4 & 16 & 3,6 & 21 & 3,9 \\
\hline 70 a 79 & 3 & 3,2 & 2 & 0,5 & 5 & 0,9 \\
\hline 80 a 89 & 1 & 1,1 & 0 & 0 & 1 & 0,2 \\
\hline \multicolumn{7}{|l|}{ Escolaridade } \\
\hline Analfabeto & 1 & 1,1 & 9 & 2,1 & 10 & 1,9 \\
\hline Fundamental Inc. & 24 & 26,4 & 164 & 37,6 & 188 & 35,7 \\
\hline Fundamental & 17 & 18,7 & 128 & 29,4 & 145 & 27,5 \\
\hline Médio & 38 & 41,8 & 120 & 27,5 & 158 & 30,0 \\
\hline Superior & 11 & 12,1 & 15 & 3,4 & 26 & 4,9 \\
\hline \multicolumn{7}{|l|}{ CAPS ad llha } \\
\hline \multicolumn{7}{|l|}{ Idade } \\
\hline 0 a 9 & 0 & 0 & 1 & 0,2 & 1 & 0,2 \\
\hline 10 a 19 & 10 & 8,8 & 28 & 5,2 & 38 & 5,8 \\
\hline 20 a 29 & 30 & 26,3 & 160 & 29,5 & 190 & 29,0 \\
\hline 30 a 39 & 37 & 32,5 & 164 & 30,3 & 201 & 30,6 \\
\hline 40 a 49 & 22 & 19,3 & 120 & 22,1 & 142 & 21,6 \\
\hline 50 a 59 & 12 & 10,5 & 59 & 10,9 & 71 & 10,8 \\
\hline 60 a 69 & 3 & 2,6 & 9 & 1,7 & 12 & 1,8 \\
\hline 70 a 79 & 0 & 0 & 1 & 0,2 & 1 & 0,2 \\
\hline 80 a 89 & 0 & 0 & 0 & 0 & 0 & 0 \\
\hline \multicolumn{7}{|l|}{ Escolaridade } \\
\hline Analfabeto & 2 & 1,8 & 7 & 1,3 & 9 & 1,4 \\
\hline Fundamental Inc. & 44 & 38,9 & 218 & 41,0 & 262 & 40,6 \\
\hline Fundamental & 25 & 22,1 & 150 & 28,2 & 175 & 27,1 \\
\hline Médio & 35 & 31,0 & 136 & 25,6 & 171 & 26,5 \\
\hline Superior & 7 & 6,2 & 21 & 3,9 & 28 & 4,3 \\
\hline
\end{tabular}

\section{Desenho do Estudo}

É um estudo descritivo, observacional, transversal realizado nos CAPSs ad, mediante a consulta ao banco de dados eletrônico da PMF.

\section{População}

Todos os usuários assistidos nos CAPS ad Continente e CAPS ad llha do Município de Florianópolis no período de 1 de julho a 31 de dezembro de 2011. 


\section{Instrumentos}

Inventário sociodemográfico e clínico. O preenchimento deste inventário foi conduzido através dos registros dos atendimentos realizados pelos profissionais que atuam no CAPS ad da PMF, arquivados em sistema eletrônico próprio. A consulta ao banco de dados digital foi feita por estudantes de Medicina, Psicologia, Enfermagem e Serviço Social, após treinamento apropriado e sob a supervisão de técnicos da PMF e docentes da UFSC. A priori, as variáveis sociodemográficas incluíram a faixa-etária, o gênero, a escolaridade, a ocupação profissional e o nível socioeconômico, sendo que as variáveis clínicas se restringiram aos principais diagnósticos relacionados ao uso de substâncias psicoativas.

\section{Análise dos Dados}

Foi conduzida uma análise descritiva com os principais elementos sociodemográficos e clínicos, procedendo-se incialmente o cálculo de proporções. Comparações entre grupos foram conduzidas, utilizando-se o qui-quadrado e o correspondente cálculo do valor de $p$.

\section{Ética em Pesquisa}

O projeto seguiu as recomendações da Resolução MS/CNS 466/2012 e foi aprovado pelo Comitê de Ética em Pesquisa com Seres Humanos da UFSC (CEPSHUFSC).

\section{Resultados}

Inicialmente foi conduzido um exame dos dados disponíveis no sistema eletrônico de atendimentos feitos pelos técnicos em saúde da PMF, assim como do formato de registro dessas informações. A seguir, produziu-se um formulário que procurou acessar a idade (por faixas etárias), o gênero, a escolaridade (por categorias) e os principias diagnósticos relacionados ao uso de álcool e outras drogas. As variáveis ocupação profissional, com grande diversidade de categorias, e nível socioeconômico foram excluídas do levantamento. Em função do formato digital dos registros, não foi possível o cálculo da média de idade, assim como da média dos anos de escolaridade.

A população examinada foi composta de 535 usuários do CAPS ad Continente e de 656 usuários do CAPS ad llha. A composição sociodemográfica foi bastante semelhante entre os CAPS ad Continente e CAPS ad Ilha (Tabela 1), tanto em relação ao padrão etário ( $77,4 \%$ vs. $81,2 \%$ : entre 20 e 49 anos de idade) quanto à escolaridade $(65,1 \%$ vs. $69,1 \%$ : até o ensino fundamental) e sobretudo em relação ao gênero ( $82,6 \%$ em ambos: sexo masculino). No entanto, quando se avaliou a escolaridade por gênero, no CAPS ad Continente as mulheres tiveram uma escolaridade significativamente mais alta do que os homens $(p=0,001)$.

No que diz respeito aos diagnósticos relacionados ao uso de substâncias psicoativas, os mais prevalentes nos CAPS ad Continente e CAPS ad Ilha foram, respectivamente:

- Dependência à cocaína/crack: $40,7 \%$ e $50,7 \%$.

- Dependência ao álcool: $28,0 \%$ e $32,97 \%$.

- Uso nocivo de álcool: $1,8 \%$ e 4,1\%.

- Uso nocivo de cocaína/crack: 0,6\% e 3,2\%.

A dependência à maconha representou uma proporção muito pequena de usuários atendidos em ambos os CAPSs ad (em torno de 2,5\%). Não foi possível precisar o diagnóstico de dependência a múltiplas substâncias ou a outras substâncias, uma vez que houve, em proporção variada nos dois CAPSs ad, superposição desse diagnóstico com os demais diagnósticos restritos a um único tipo de substância, razão pela qual se optou por excluí-lo da análise.

No que diz respeito ao gênero, a relação entre homens e mulheres em ambos os CAPSs ad apresentou a seguinte relação, considerando que, para cada mulher nos CAPS ad Continente e CAPS ad Ilha, respectivamente, tivemos:

- Dependência à cocaína/crack: 8 e 6,2 homens.

- Dependência ao álcool: 8,5 e 5,5 homens.

No CAPS ad llha, entre os usuários com dependência ao álcool, as mulheres eram mais jovens do que os homens, com um predomínio feminino na faixa etária abaixo dos 40 anos de idade (20,9\% vs. $12 \%$; $p=0,075)$. No CAPS ad Continente, esse resultado não se repetiu (Figuras 1 e 2). Também não houve diferença de gênero acerca da idade de apresentação, em ambos os serviços, entre usuários dependentes de cocaína/crack. Em outras palavras, homens e mulheres dependentes de cocaína/ crack não diferiram em relação à idade.

Contudo, ao se comparar a idade de apresentação entre usuários dependentes de álcool e usuários dependentes de cocaína/crack em cada sexo, observouse um nítido predomínio de idades mais baixas entre os usuários dependentes de cocaína/crack. Em outras palavras, usuários dependentes de cocaína/ crack tenderam a se apresentar mais jovens do que os usuários dependentes de álcool, em ambos os serviços (CAPS ad Continente: feminino: $p=0,017$, masculino: $p<0,001$; CAPS ad llha: feminino: $p=0,002$, masculino: $p<0,001)$. 


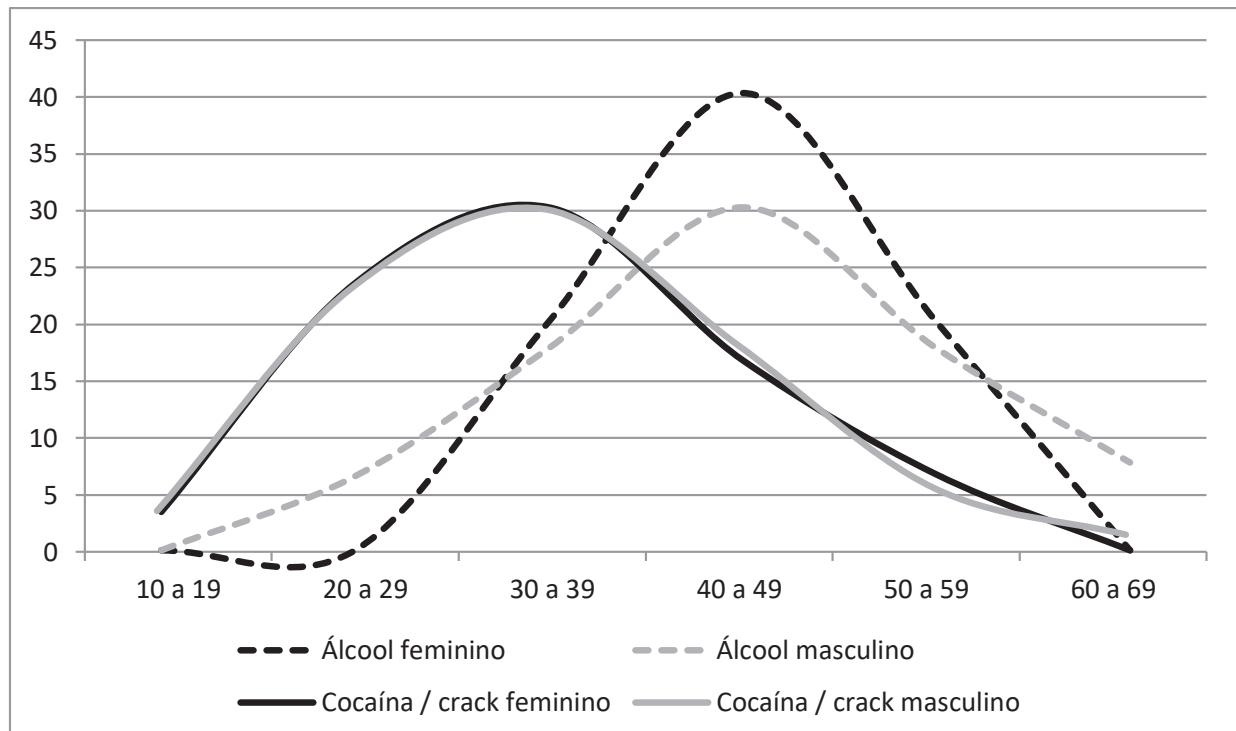

Álcool feminino vs. Cocaína/crack feminino: $X^{2}=8,1 ; p=0,017$; Álcool masculino vs. Cocaína/ crack masculino: $X^{2}=53,7 ; p<0,001$

Figura 1 - Proporção de usuários, por dependência às substâncias psicoativas, em relação à idade e gênero, no CAPS ad Continente

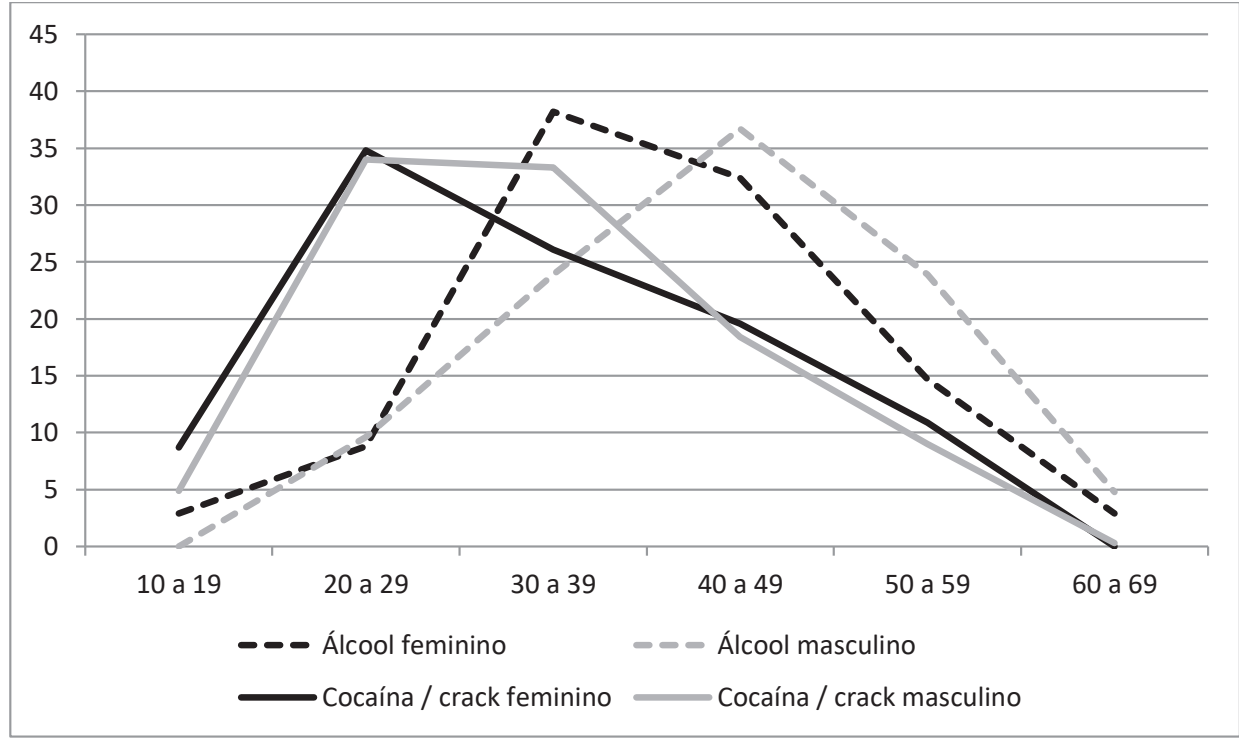

Álcool feminino vs. Cocaína/crack feminino: $X^{2}=9,4 ; p=0,002$; Álcool masculino vs. Cocaína/ crack masculino: $X^{2}=77,9 ; p<0,001$; Álcool feminino vs. Álcool masculino: $X^{2}=3,16 ; p=0,075$

Figura 2 - Proporção de usuários, por dependência às substâncias psicoativas, em relação à idade e gênero, no CAPS ad llha

\section{Discussão}

O presente estudo observou que, entre os usuários assistidos nos CAPSs ad de Florianópolis, os diagnósticos mais prevalentes foram dependência à cocaína/crack e dependência ao álcool, com uma razoável superioridade do primeiro, sendo que o gênero masculino teve um predomínio expressivo em ambos os diagnósticos. Observou-se também que, entre os usuários dependentes de álcool do CAPS ad Ilha, as mulheres eram mais jovens do que os homens e, em ambos os CAPSs ad, os usuários dependentes de cocaína/crack eram mais jovens do que os usuários dependentes de álcool. 
Ao se comparar as prevalências de dependência de cocaína/crack e dependência de álcool na população brasileira presente na comunidade, $0,9 \%{ }^{(11)}$ e $9-12,3 \%{ }^{(12-}$ ${ }^{13)}$, respectivamente, com as proporções desses dois diagnósticos nos dois CAPSs ad avaliados, observase claramente uma importante inversão em favor do primeiro diagnóstico. Em outras palavras, enquanto que na comunidade, para cada usuário dependente de cocaína/crack, há aproximadamente 10 usuários dependentes de álcool (1 para 10), nos dois CAPSs ad essa relação fica em torno de 1 para 0,6 (os dados comunitários citados acima são relativos apenas à dependência à cocaína, não havendo informação sobre dependência ao crack). Várias hipóteses poderiam explicar essa significativa inversão, embora sem avaliação no presente estudo e com pouquíssima comprovação por estudos prévios. No que diz respeito à organização dos serviços assistenciais, estaria havendo um direcionamento preferencial dos usuários de álcool para outros serviços tradicionais da saúde (unidade básica de saúde, hospital) ou para as comunidades terapêuticas? A expressiva presença das Equipes da Saúde da Família na atenção básica de Florianópolis, associada à presença dos Núcleos de Apoio à Saúde da Família nos cinco distritos sanitários ${ }^{(14)}$, estaria contribuindo para o acesso preferencial dos usuários de álcool neste nível de atenção? Por outro lado, a aplicação das "intervenções breves" para os problemas relacionados ao uso de álcool na atenção primária, conforme orientação da Organização Mundial de Saúde ${ }^{(15)}$, em que os casos de dependência ao álcool seriam preparados e devidamente encaminhados para a atenção especializada, estaria deixando de ser realizada? O maior estigma ao usuário de cocaína/ crack pelos profissionais de saúde da atenção básica, se realmente presente, poderia estar influenciando este fluxo diferenciado entre os usuários de álcool e os usuários de cocaína/crack na rede de atenção à saúde? Outra variável de destaque e essencial na orientação do fluxo dos casos na Saúde Mental do SUS, a gravidade, poderia explicar a maior presença dos usuários de cocaína/crack nos CAPSs ad se confirmada a percepção da sociedade de que estes seriam mais graves do que os usuários de álcool? Contudo, um estudo espanhol com usuários em tratamento encontrou maior gravidade entre os usuários de álcool quando comparados aos usuários de cocaína em vários parâmetros médicos e psicossociais $^{(16)}$. Em relação à hospitalização, observouse um predomínio dos transtornos mentais pelo uso de álcool sobre os decorrentes do uso de múltiplas substâncias ou de outras substâncias psicoativas (muito provavelmente decorrentes principalmente de cocaína/ crack), nos dados referentes à internação hospitalar no
Estado do Rio de Janeiro (período de 2008 a 2010)(17), $14,3 \%$ vs. $9,0 \%$, e à internação psiquiátrica em Campo Grande, MS (de 2004 a 2008)(18), 24,6\% vs. 5,9\%, respectivamente (as taxas descritas do Estado do Rio de Janeiro se referem ao sexo masculino, uma vez que no sexo feminino as taxas são muito próximas). Observase então uma relação entre usuários dependentes de cocaína/crack e usuários dependentes de álcool internados em torno de 1 para 1,5 a 4. Transpondo esta realidade para Florianópolis, poderíamos dizer que a internação dos usuários dependentes de álcool, em comparação com a dos usuários dependentes de cocaína/crack, não explicaria a inversão destes dois diagnósticos no presente estudo. A despeito de todas estas considerações sobre a estruturação da rede de atenção à saúde, há ainda a autopercepção dos usuários problemáticos de álcool envolvendo as dificuldades de reconhecimento do próprio problema e da necessidade de procura por tratamento que poderia estar levando à redução de sua presença nos serviços assistenciais. Dados epidemiológicos nacionais dos Estados Unidos da América apontam que menos de $25 \%$ destes usuários já se submeteram a tratamento por tais problemas ${ }^{(19)}$. Em suma, diante de tamanha complexidade, faz-se necessário o levantamento de mais informações para o melhor entendimento do caminho que os usuários problemáticos de álcool e cocaína/crack percorrem da comunidade para os serviços assistenciais.

As diferenças de gênero, com predomínio de homens entre os dois principais diagnósticos em ambos os CAPSs ad e idade mais jovem entre as mulheres usuárias dependentes de álcool no CAPS ad Ilha, corroboram achados prévios e contribuem para a compreensão da apresentação desigual desses problemas entre homens e mulheres. Embora o padrão de uso de drogas venha se modificando entre as mulheres nas últimas décadas, ainda é notável o predomínio de homens dependentes de álcool e cocaína em nosso meio, segundo levantamentos nacionais comunitários que apontam uma razão homem : mulher de 5:1 e 3:1, respectivamente ${ }^{(11-12)}$. Ademais, em estudo realizado em Florianópolis com população adulta, observaram-se nos homens três vezes mais "problemas pelo uso de álcool" do que nas mulheres ${ }^{(20)}$. Especificamente em relação aos usuários de crack, conforme levantamento nacional realizado em "cenas de uso" (Florianópolis é um dos sítios de estudo), novamente um predomínio masculino, com uma razão próxima de 3,5:1(21). Contudo, no presente estudo, que apresenta resultados de gênero muito similares aos estudos feitos nos CAPSs ad de Piauí, Curitiba e Blumenau $^{(7-8,22)}$, há que se destacar proporcionalmente 
a menor presença das mulheres nos serviços, quando comparada à comunidade. Apenas no CAPS ad llha a relação de gênero ficou muito próxima da comunidade, indicando possíveis e reais características das mulheres desta área de abrangência e/ou da organização do serviço (por exemplo, a criação de um grupo específico para mulheres), as quais poderiam ter levado à maior presença feminina nesse CAPS ad. Nesse aspecto, se por um lado, de maneira geral, buscam mais ajuda, as mulheres são mais estigmatizadas socialmente pela dependência às drogas, com menor probabilidade de serem diagnosticadas por problemas pelo uso de álcool e apresentando obstáculos ao tratamento, tais como medo do abandono do cônjuge/parceiro e medo de perda dos filhos ${ }^{(23)}$.

No que diz respeito à idade mais jovem das mulheres usuárias dependentes de álcool no CAPS ad llha, esse resultado provavelmente reproduz o que ocorre na comunidade (os dados mundiais são conflitantes) e, em parte, poderia estar associado à maior sensibilidade das mulheres aos efeitos do álcool, levando precocemente à busca por ajuda e/ou ao surgimento de problemas ${ }^{(24)}$.

Prosseguindo nas diferenças de idade do presente estudo, o resultado demonstrando a idade mais avançada dos usuários dependentes de álcool comparados aos usuários dependentes de cocaína/ crack, em ambos os CAPSs ad, corrobora os resultados encontrados em estudo espanhol que também observou maior gravidade entre os primeiros ${ }^{(16)}$. Embora sejam imprecisos neste aspecto etário, os dados nacionais comunitários mostram uma razão proporcionalmente maior de dependência sobre o uso no último ano (prevalência de dependência : prevalência de uso no último ano), entre os usuários de cocaína comparados aos usuários de álcool(11-13). Em outras palavras, a maior progressão para a dependência entre os usuários de cocaína, e muito provavelmente em menor tempo, talvez possa explicar essa diferença de idade, que estaria amplificada, em decorrência dos mesmos motivos, pela presença dos usuários de crack nos dois serviços.

As principias limitações deste estudo se referem à forma digital de coleta de dados, limitando ou impedindo a análise de variáveis de interesse, e ao procedimento de inclusão dos principais diagnósticos que levou à dificuldade de distinguir claramente os usuários apenas dependentes de álcool e os usuários apenas dependentes de cocaína/crack dos usuários com diagnóstico de dependência múltipla, assim como diferenciar os usuários de cocaína dos usuários de crack.

\section{Conclusão}

As principais contribuições do presente estudo foram apresentar o perfil de usuários de CAPSs ad, caracterizado pelo predomínio acentuado de homens e discreto de usuários de cocaína/crack em relação aos usuários de álcool, apresentação de usuários de álcool mais jovens entre as mulheres do que os homens e de usuários de cocaína/crack mais jovens do que usuários de álcool. Dessa forma, procurou lançar luz sobre determinados aspectos que influenciam tanto a morbidade quanto o fluxo de usuários de álcool e outras drogas dentro da rede de atenção, em especial no Município de Florianópolis.

\section{Agradecimentos}

Aos técnicos da PMF e estudantes da UFSC que compuseram a equipe PET e contribuíram para a coleta de dados e aos usuários dos CAPSs ad de Florianópolis.

\section{Referências}

1. Ministério da Saúde (BR). Portaria GM/MS no. 336, de 19 de fevereiro de 2002. Estabelece que os Centros de Atenção Psicossocial poderão constituir-se em modalidades de serviço. Brasília; 2002.

2. Ministério da Saúde (BR). Saúde Mental no SUS: as novas fronteiras da Reforma Psiquiátrica. Relatório de Gestão 2007-2010. Brasília; 2011.

3. Ministério da Saúde (BR). Portaria GM/MS no. 3.088, de 23 de dezembro de 2011. Institui a Rede de Atenção Psicossocial para pessoas com sofrimento ou transtorno mental e com necessidades decorrentes do uso de crack, álcool e outras drogas, no âmbito do Sistema Único de Saúde. Brasília; 2011.

4. Ministério da Saúde (BR). Secretaria de Atenção à Saúde, SVS/CN-DST/AIDS. A Política do Ministério da Saúde para a Atenção Integral a Usuários de Álcool e outras Drogas. Brasília; 2003.

5. Presidência da República (BR). Decreto no. 7.179, de 20 de maio de 2010. Institui o Plano Integrado de Enfrentamento ao Crack e outras Drogas. Brasília; 2010. 6. Silva CC, Costa MCO, Carvalho RC, Amaral MTR, Cruz NLA, Silva MR. Iniciação e consumo de substâncias psicoativas entre adolescentes e adultos jovens de Centro de Atenção Psicossocial Antidrogas/CAPS-AD. Ciên Saúde Coletiva. 2014;19:737-45.

7. Monteiro CFS, Fé LCM; Moreira MAC, Albuquerque IEM; Silva MG, Passamani MC. Perfil sociodemográfico e adesão ao tratamento de dependentes de álcool em CAPS-ad do Piauí. Esc Anna Nery. 2011; 15:90-5. 
8. Faria JG, Schneider DR. O perfil dos usuários do CAPSad-Blumenau e as políticas públicas em saúde mental. Psicol Soc. 2009;21:324-33.

9. Galduróz JCF, Noto AR, Nappo SA, Carlini EA. Use of psychotropic drugs in Brazil: household survey in the 107 biggest Brazilian cities - 2001. Rev. Latino-Am. Enfermagem. 2005;13:888-95.

10. Secretaria de Gestão do Trabalho e da Educação na Saúde (BR). Edital Conjunto no. 27, de 17 de setembro de 2010. Convidam à apresentação de propostas com vistas à seleção de projetos para participação no Programa de Educação pelo Trabalho para a Saúde - PET-Saúde/Saúde Mental-Crack, Álcool e outras Drogas. Diário Oficial da União; 2010; 17 set.

11. Abdalla RR, Madruga CS, Ribeiro M, Pinsky I, Caetano $\mathrm{R}$, Laranjeira R. Prevalence of cocaine use in Brazil: data from the II Brazilian national alcohol and drugs survey (BNADS). Addict Behav. 2014;39:297-301.

12. Laranjeira R, Pinsky I, Sanches M, Zaleski M, Caetano

R. Alcohol use patterns among Brazilian adults. Rev Bras Psiquiatr. 2010;32:231-41.

13. Fonseca AM, Galduróz JCF, Noto AR, Carlini EA. Comparison between two household surveys on psychotropic drug use in Brazil: 2001 and 2004. Ciênc Saúde Coletiva. 2010; 15:663-70.

14. Secretaria Municipal de Saúde de Florianópolis. Saúde da Família. [Internet]. http://www.pmf.sc.gov.br/entidades/ saude/index.php?cms $=$ saude + da + familia\&menu $=5$ [Acesso 24 ago 2016].

15. Babor TF, Higgins-Biddle JC. Alcohol screening and brief intervention: dissemination strategies for medical practice and public health. Addiction. 2000;95:677-86.

16. López-Goñi JJ, Fernández-Montalvo J, Arteaga A. Differences between Alcoholics and Cocaine Addicts Seeking Treatment. Span J Psychol. 2015;18:E2.

17. Pereira PK, Santos SA, Lima LA, Legay LF, Santos JFC, Lovisi GM. Transtornos mentais e comportamentais no Sistema de Informações Hospitalares do SUS (SIHSUS) no estado do Rio de Janeiro no período de 1999 a 2010. Cad Saúde Coletiva. 2012;20:482-91.

18. Souza JC, Souza N, Magna LA. Tempo médio de hospitalização e categorias diagnósticas em hospital psiquiátrico. J Bras Psiquiatr. 2008;57:112-6.

19. Hasin DS, Stinson FS, Ogburn E, Grant BF. Prevalence, correlates, disability, and comorbidity of DSM-IV alcohol abuse and dependence in the United States: results from the National Epidemiologic Survey on Alcohol and Related Conditions. Arch Gen Psychiatry. 2007;64:830-42.

20. Reisdorfer E, Büchele F, Pires ROM, Boing AF. Prevalence and associated factors with alcohol use disorders among adults: a population-based study in southern Brazil. Rev Bras Epidemiol. 2012;15:582-94.
21. Fiocruz/Ministério da Justiça (BR). Perfil dos usuários de crack e/ou similares no Brasil. Brasília; 2013.

22. Ribeiro DR, Carvalho DS. O padrão de uso de drogas por grupos em diferentes fases de tratamento nos Centros de Atenção Psicossocial Álcool e Drogas (CAPS-AD). J Bras Psiquiatr. 2015;64:221-29.

23. Brienza RS, Stein MD. Alcohol use disorders in primary care: do gender-specific differences exist? J Gen Intern Med. 2002;17:387-97.

24. Erol A, Karpyak VM. Sex and gender-related differences in alcohol use and its consequences: Contemporary knowledge and future research considerations. Drug Alcohol Depend. 2015;156:1-13. 